\title{
Formación lectora para la inclusión social. Estudio de la perspectiva educativa para los alumnos de la ESO en la Comunidad de Canarias
}

\author{
Zyuzina, Alexandra \\ Universidad de La Laguna, San Cristóbal de La Laguna, España \\ Aleksandra_az@mail.ru
}

\section{Resumen}

En la segunda mitad del siglo XX ha cambiado el paradigma social hacia el desarrollo de la personalidad y la consolidación de los valores cívicos. El Gobierno de España y el Gobierno de Canarias, destacan la finalidad de la Educación Secundaria Obligatoria: la adquisición de los elementos básicos de la cultura en sus aspectos humanístico, artístico, científico y tecnológico para su incorporación posterior en la vida personal y laboral, y para el ejercicio de sus derechos y obligaciones cívicos.

En el área de Lengua Castellana y Literatura se trata de consolidar los hábitos de la lectura para garantizar el acceso libre a las fuentes de información de diversa naturaleza para todos los miembros de la sociedad sin ningún tipo de discriminación. La adquisición de la competencia lectora contribuirá al desarrollo personal, impidiendo la exclusión social y, como una de las consecuencias, la exclusión económica. No obstante, últimamente está descendiendo el interés a la lectura. Ante el mundo de nuevas tecnologías, los profesionales debemos ofrecer a nuestros alumnos una respuesta educativa que pueda captar su atención y adaptar nuestros métodos a su realidad, para formar a unos lectores competentes bajo los conceptos del acceso a los conocimientos y la equidad ante el uso de las herramientas para lograrlo.

Así, una de las respuestas pedagógicas para el fomento de la competencia lectora es el Plan Lector que se aplica actualmente en los colegios y los institutos de Canarias. El Plan Lector pretende mejorar la comprensión lectora y fomentar el hábito de lectura. Podemos considerar el Plan Lector como una de las herramientas fundamentales para desarrollar la competencia lectora para la futura inclusión personal, social y económica.

Palabras clave: comprensión lectora, formación lectora, inclusión social, Plan Lector, ESO, Canarias.

\section{INTRODUCCIÓN}

En la segunda mitad del siglo XX ha cambiado el paradigma social hacia el desarrollo de la personalidad y la consolidación de los valores cívicos. Acorde a los nuevos objetivos se va estructurando el sistema educativo.

El Gobierno de España en general, y el Gobierno de Canarias en particular, destacan la finalidad de la Educación Secundaria Obligatoria: la adquisición de los elementos básicos de la cultura en sus aspectos humanístico, artístico, científico y tecnológico para su incorporación posterior en la vida personal y laboral, y para el ejercicio de sus derechos y obligaciones cívicos.

En el área de Lengua Castellana y Literatura, según el Currículo, se trata de consolidar los hábitos de la lectura para garantizar el acceso libre a las fuentes de información de diversa naturaleza para todos los miembros de la sociedad, sin ningún tipo de discriminación. Como consiguiente, vemos que la inclusión social es uno de los objetivos fundamentales del sistema educativo actual en España. La adquisición de la competencia lectora contribuirá al desarrollo personal, impidiendo la exclusión social y, como una de las consecuencias, la exclusión económica

Siguiendo las ideas de Mendoza (1998) definimos la lectura como «una compleja actividad de conocimiento, en la que interviene el conjunto de dominios, destrezas y habilidades lingüísticas (facetas de la pragmática comunicativa), de conocimientos (enciclopédicos, lingüísticos, paralingüísticos, metatextuales e intertextuales), de la misma experiencia extralingüística que posea el lector (convencionalismos sociales y culturales, ideologías, situaciones, etc.) a los que se alude de maneraimplícita o explícita en el texto» (p. 49). 


\section{DEFINICIÓN DE CONCEPTOS: OMPRENSIÓN LECTORA, FORMACIÓN LECTORA Y LA MOTIVACIÓN Y ANIMACIÓN A LA LECTURA}

Consideramos la lectura como una de las actividades diarias que nosotros realizamos durante toda la vida y que forma parte fundamental del aprendizaje de todo tipo. A continuación exponemos los conceptos incluidos en el proceso de la adquisición de dicho aprendizaje.

\subsection{Sobre la comprensión de lectura}

Compartimos la idea de González Fernández (2004) de que «la lectura es el mecanismo más importante de transmisión de conocimientos en las sociedades cultas actuales. Ser experto en esta destreza es fundamental en todas las etapas del sistema educativo. En la etapa de la educación secundaria obligatoria la adquisición de conocimientos está condicionada (entre otros muchos factores) por el nivel de comprensión lectora alcanzado por el sujeto, en definitiva, por su capacidad para aprender a partir de los textos» (p. 15).

Para que el proceso de aprendizaje llegue a su fin, tenemos que garantizar el cumplimiento de múltiples condiciones para la realización eficaz de la lectura.Los objetivosesenciales de lectura son la comprensión y la interpretación. Evidentemente, la comprensión depende de la finalidad de lectura.

Sánchez Miguel (2010) destaca cuatro finalidades principales: «1) lectura para fines privados; 2) lectura para fines públicos; 3) lectura para fines profesionales; 4) lectura para fines educativos» (p. 64). Asimismo, la forma de lectura depende del objetivo del lector. El lector debe poseer conocimientos de tipo muy variado para poder abordar con éxito su lectura, como, por ejemplo, los conocimientos paralingüísticos: elementos tipográficos, separación de palabras (sílabas, párrafos, etc.), la organización de la información (índice, prologo, etc.); conocimientos morfológicos, sintácticos y semánticos; conocimientos sobre el mundo, etc. (Colomer y Camps, 1996, p. 56 - 63).

Entendiendo que la lectura es un proceso complejo y, para que el aprendizaje de los alumnos sea significativo, hay que enseñar a comprender la lectura, que supone instruir explícitamente a los alumnos para que sean ellos quienes generen objetivos de lectura, supervisen si están comprendiendo, identifiquen el esquema retórico del texto o se pregunten que saben ya del tema (Sánchez Miguel, 2010, p. 90).

\subsection{La formación lectora}

Dentro del período de escolarización podemos destacar dos etapas de la formación lectora.En general, el objetivo de este proceso es la formación de un lector competente. Para cumplir con éxito dicha tarea tenemos que ser conscientes de que las personas implicadas en este proceso, los familiares, los profesores y demás personas, deben tener en cuenta todas aquellas condiciones que permitan satisfacer las necesidades del lector y llevarlo a un aprendizaje significativo.

El lector debe dominar todas aquellas características que posee el texto y tener el objetivo de lectura establecido con claridad. Una de las ideas fundamentales es que la lectura le da acceso a múltiples conocimientos sobre el mundo.

Es importante implantar en los alumnos la idea del aprendizaje significativo a través de la lectura. Estamos de acuerdo con Pérez Gómez (véase Gimeno Sacristán, 2008) en que «los seres humanos aprenden de forma relevante cuando adquieren significados que consideran útiles para sus propósitos vitales» (p. 72). Dado que reconocemos la formación lectora como un proceso de aprendizaje, compartimos la idea de Pérez Gómez de que en el aprendizaje están implicados factores cognitivos y metacognitivos, factores motivacionales y emotivos, y factores sociales y culturales.

Teniendo en cuenta lo arriba indicado, podemos destacar dos aspectos de la formación lectora: la adquisición de las competencias básicas y la motivación o la animación a la lectura. Más adelante prestaremos atención especial al fenómeno de la motivación o la animación a la lectura en la formación lectora con el objetivo de formar lectores competentes. 


\subsection{La motivación y la animación a la lectura}

El estudio de la motivación es un ámbito de estudio muy popular últimamente. En nuestra consideración, todo tiene su explicación. ¿Por qué llegamos a este problema? Falta de motivación por parte de los alumnos. Pensamos que es un problema grave de la sociedad. No es únicamente el problema del docente que no puede hacer sus clases divertidas, sino es una consecuencia de la sociedad desmotivada.

La motivación es el motor del aprendizaje, y la motivación se encuentra influida por las emociones, las creencias, los intereses y los valores. Cada lector es portador de sus intereses y sus características particulares, tanto personales dadas, como adquiridas a lo largo de la vida.

Colomer y Camps (1996) subrayan la influencia de la actitud ante la lectura y lo destacan diciendo que «para que se llegue a un buen hábito lector, es necesario que el niño se lo pase bien leyendo. Para conseguir buenos lectores, capaces de asimilar la información que les proporciona la lectura y hacer un uso creativo de ella, es esencial la actitud emocional que se cree ante la misma. Por eso, el niño ha de sentir que la lectura forma parte de la vida, que es un medio extraordinario de comunicación y de información» (p. 22).

La actitud del adulto ante los libros puede ser también muy estimulante. La calidez que dé a una lectura, el gusto con que hable de un autor, la periodicidad a la que acostumbre a sus alumnos a escuchar una lectura bien hecha, con un tono afectuoso, íntimo, hará que ellos esperen estos momentos y respeten los libros como fuentes de placer y de información. El clima que envuelva la lectura será, sin duda, una gran motivación para querer aprender a leer (Colomer y Camps, 1996, p. 22).

El concepto de la motivación se relaciona con el hecho de que los alumnos vean que la lectura tiene sentido. En este aspecto ayuda la claridad de los objetivos de lectura. Los objetivos de lectura están vinculados con diferentes situaciones de la vida, no únicamente con el mundo escolar. Podemos contar, asimismo, con la curiosidad natural de los niños. Es muy importante desarrollar el hábito lector en la infancia, puesto que, con un manejo adecuado, en la mayoría de los casos se queda para toda la vida.

En la animación a la lectura nos ayudan los mismos componentes y procesos de lectura: anticipación, descodificación, comprensión, interpretación. La anticipación es la actividad que desarrolla el lector inmediatamente antes de dar curso a la descodificación del texto. Esta actividad corresponde a la previsión, en gran medida intuitiva, sobre el significado global y sobre la tipología textual. [...] Gracias a la anticipación, se construye un marco inicialmente provisional que pone en marcha la intuición sobre las probabilidades temáticas, estilísticas, de intencionalidad, de orientación, etc. que puede aportar un determinado texto (Mendoza, 1998, p. 98 - 99).

Para concluir queremos destacar algunos aspectos que nos pueden servir de ayuda para motivar a los alumnos y animarlos a la lectura:

1. Reconocer la personalidad de cada alumno, sus características personales, culturales, sociales, lingüísticas, etc.

2.Ser ejemplo constante de valoración de lectura como fuente de información y disfrute de la lectura y de la lengua escrita.

3. Hacer que los libros estén a disposición de los alumnos.

4.Planificar tareas y consolidar los objetivos de la lectura para los alumnos.

5. Implantar la idea del privilegio de ser lector competente.

6. Divulgar el concepto de la utilidad de lectura para resolver múltiples tareas cotidianas.

7.Dar a los alumnos la posibilidad de escoger las lecturas de su interés y que aprendan a argumentar su selección.

8. No imponer la literatura oficial sino guiar por el mundo de la literatura para que se forme en ellos el gusto por la literatura.

9. Aprovechar de los conocimientos previos y las habilidades de los alumnos.

10. Fomentar la aplicación de sus destrezas particulares en el proceso de lectura o en la selección de libros. 


\section{LA REALIDAD DE LA LECTURA EN LA ESO EN CANARIAS}

Aunque se está reforzando el reconocimiento de la educación literaria, últimamente está descendiendo el interés y la animación a la lectura. Ante el mundo de nuevas tecnologías, los profesionales debemos ofrecer a nuestros alumnos una respuesta educativa que pueda captar su atención y adaptar nuestros métodos a su realidad, para alcanzar nuestro objetivo de formar a unos lectores competentes bajo los conceptos del acceso a los conocimientos y la equidad ante el uso de las herramientas para lograrlo.

\subsection{Aproximación a la realidad social y educativa}

Actualmente, la competencia lectora y el hábito lector se quedaron fuera de la lista de las competencias clave. Aunque, sin lugar a dudas, persiste dentro de todas las que se desarrollan durante la escolarización. Con la multitud de recursos y actividades que la actualidad ha incorporado a la vida social, la lectura se quedó en cierto olvido y lo entendemos como uno de los problemas de la sociedad moderna.

Sánchez Miguel (2010) destaca que «la adquisición de la competencia lectora es un complejo de hábitos, competencias y habilidades lingüísticas» (p. 66-70):

1. Un buen cuerpo de conocimientos para poder hacer inferencias, para asignar roles correctos a los distintos elementos de las proposiciones, para relacionar distintas partes del discurso.

2. La habilidad para reconocer palabras con precisión y rapidez.

3. Competencia retórica, la capacidad para detectar, interpretar y usar ciertos recursos textuales (anáforas, marcadores retóricos, elementos que avisan de la introducción de resúmenes o recapitulaciones...) que sirven para orientar el proceso de lectura.

4. Habilidades metacognitivas para poder regularse durante la lectura, marcándose objetivos y detectando/solucionando cualquier problema de comprensión que amenace la consecución de los mismos.

\subsection{Plan Lector en la Comunidad Canaria}

Una de las respuestas pedagógicas para el fomento de la competencia lectora es el Plan Lector (Gobierno de Canarias. Plan de Lectura y Bibliotecas Escolares de Canarias), un programa que se aplica actualmente en los centros, los colegios y en los institutos de Canarias. El Plan Lector pretende mejorar la comprensión lectora y fomentar el hábito de lectura, con el objetivo de incidir en el rendimiento y en la formación del alumnado en distintos ámbitos. A raíz de este planteamiento, podemos considerar el Plan Lector como una de las herramientas fundamentales para desarrollar la competencia lectora, una de las competencias clave para la futura inclusión personal, social y económica.

El Plan Lector es un instrumento complementario de los centros educativos, es evaluable por parte de todo el profesorado, y su actuación será fundamental para el correcto desarrollo ya que la lectura constituye un factor importante para el desarrollo de las competencias.

El objetivo primordial del Plan Lector es el de fomentar y consolidar el hábito lector, puesto que leer habitualmente es la actividad que mejor puede incidir en el rendimiento y en la formación del alumnado.

El Plan Lector se centra principalmente en el desarrollo de las siguientes competencias básicas:

a. Competencia en Comunicación Lingüística: mediante el uso del lenguaje de forma competente en distintas esferas de la vida social, la utilización de diferentes tipos de textos y sus estructuras y el fomento de la lectura de diferentes obras literarias así como el fomento de la escritura siguiendo modelos.

b. Competencia social y ciudadana: mediante el uso del lenguaje como medio eficaz de transmisión de valores de convivencia, respeto y entendimiento entre las personas. Y el análisis de los usos discriminatorios del lenguaje para la erradicación de prejuicios y estereotipos. 
c. Competencia para aprender a aprender: se logra con el uso del lenguaje como instrumento de acceso al saber, de representación del mundo y de construcción de conocimientos. Así como la reflexión sobre la lengua (sus conceptos y procedimientos) y su aplicación a las propias producciones (aprender a aprender la lengua). Y la elaboración de resúmenes y esquemas.

d. Autonomía e iniciativa personal: se desarrolla a través de un uso del lenguaje como medio fundamental para la toma de decisiones. El diálogo como forma de regulación y orientación de la propia actividad. Autoevaluación crítica del desarrollo de las actividades grupales e individuales. Y el desarrollo de cualidades personales.

Los objetivos del funcionamiento del Plan Lector resultan evidentes. La siguiente etapa consiste en la selección de lecturas para el alumnado de cada curso. En primer lugar el Consejo Escolar se basa en los currículos educativos para cada nivel de enseñanza. Se trata de elegir entre la multitud de ejemplos de la literatura infantil juvenil. Aquí nos gustaría plantear una cuestión: la posibilidad de compaginar la literatura actual con la literatura clásica. En nuestra consideración, el desarrollo del hábito lector sería imposible sin la formación del buen gusto literario basado en el nivel de la calidad literaria. La riqueza del fondo de la literatura clásica universal nos permite elegir las obras que consideremos más relevantes para cada curso atendiendo a las características de cada grupo y al mismo tiempo dificulta el proceso de selección. En este lugar podemos reflexionar sobre las posibles alternativas.

Para concluir, cabe destacar el hecho de que una de las condiciones del funcionamiento del Plan Lector es la contribución de la familia al fomento de la lectura en sus hijos. Tanto la escuela como la familia tienen influencias en la educación de nuestros alumnos, puesto que las responsabilidades están compartidas entre ambas instituciones. A raíz de este planteamiento, debemos considerar que padres y madres y el profesorado deben cooperar en la educación integral del alumnado, procurando establecer relaciones y trabajar de forma colaborativa, coordinada para un único fin, compartiendo responsabilidades en la enseñanza de valores, normas y costumbres, en el desarrollo de habilidades para la vida, en la educación en destrezas básicas para el aprendizaje y en la transmisión de normas y responsabilidades.

\section{CONCLUSIONES}

La lectura nos acompaña desde que somos pequeños y se considera una de las herramientas fundamentales de aprendizaje, una fuente interminable de información y de conocimiento de todo tipo. Parece una paradoja, que pasamos por una crisis lectora, un momento en que, lamentablemente, está perdido el hábito lector y el interés por la lectura entre los niños y los adolescentes. Las nuevas tecnologías y las redes sociales han sustituido la lectura en muchos casos.

Actualmente debemos consolidar los esfuerzos en el fomento de la lectura y en la formación de lectores competentes. En el proceso de la educación están implicados varios componentes: la familia, la escuela y otras personas que pueden influir significativamente en las ideas y el sistema de valores y conocimientos del alumnado.

Al mismo tiempo, podemos afirmar, basándonos en múltiples ejemplos positivos, que la lectura sigue teniendo su importancia y su valor en la vida actual. Muchos niños y adolescentes reconocen el papel fundamental del libro en su vida y en la educación y expresan las opiniones que coinciden con la idea de Delgado (2004) de que «[...] la lectura, en efecto, recogerá los sucesos que acontecen a determinados personajes en un espacio y en un tiempo. Pero las obras literarias también contienen y proyectan valores morales, conducen a una sensibilidad estética y ofrecen un cúmulo de conocimientos de realidades verificables, imprevistas o desconocidas» (p. 7-16).

Queda claro que no solamente tenemos que seguir apoyando la metodología existente que ha demostrado ya su eficacia en la formación lectora, sino también buscar nuevos caminos para el desarrollo en los niños y en los adolescentes en la etapa de la escolarización, de las habilidades que les permitirán adquirir nuevos conocimientos y ser lectores competentes y miembros activos de la sociedad moderna sin exclusión de ningún tipo. 


\section{REFERENCIAS BIBLIOGRÁFICAS}

Colomer, A. Camps (1996). Enseñar a leer, enseñar a comprender. Madrid, Celeste/MEC, Madrid.

Delgado, J. J. (2004). «Prólogo», en Antología. Los mejores relatos canarios del siglo XX. Alfaguara, Madrid, pp.7-16.

Gobierno de Canarias. «Plan de Lectura y Bibliotecas Escolares de Canarias» (s.f.).

Gimeno Sacristán, J. (comp.) (2008). Educar por competencias, ¿qué hay de nuevo? Morata, Madrid.

González Fernández, A. (2004). Estrategias de comprensión lectora. Madrid, Síntesis. Mendoza Fillola, A. (1998). Tú, lector. Aspectos de la interacción texto-lector. Barcelona, Octaedro.

Sanchez Miguel, E. (2010). La lectura en el aula. Que se hace, que se debe hacer y que se puede hacer. Barcelona, Graó. 